\title{
Hamiltonian formalism of bimetric gravity in vierbein formulation
}

\author{
Josef Klusoñ ${ }^{\mathrm{a}}$ \\ Department of Theoretical Physics and Astrophysics, Faculty of Science, Masaryk University, Kotlářská 2, 61137 Brno, Czech Republic
}

Received: 26 May 2014 / Accepted: 15 July 2014 / Published online: 8 August 2014

(C) The Author(s) 2014. This article is published with open access at Springerlink.com

\begin{abstract}
This paper is devoted to the Hamiltonian analysis of bimetric gravity in the vierbein formulation. We identify all constraints and determine their nature. We also show the existence of an additional constraint so that the scalar mode can be eliminated.
\end{abstract}

\section{Introduction}

Bimetric theories of gravity are based on the idea of joining the two tensors $\hat{g}_{\mu \nu}$ and $\hat{f}_{\mu \nu}$ in a symmetric way when each tensor has its own Einstein-Hilbert action and then couple these actions through a non-derivative mass term. The presence of this term reduces the separate coordinate invariances to a single one $[1,2] .{ }^{1}$ If we set one metric as the background metric without any dynamics we find that the bimetric theory is reduced to a single metric massive gravity theory with a mass term that at the linear limit leads to the Fierz-Pauli free theory [3]. However, it was shown soon that this theory propagates ghost modes at non-linear level $[4,5]$. On the other hand, a new form of the massive term was proposed recently in $[6,7,10,11]$, which was shown to be ghost free even at the non-linear level [9, 12]; see also [13,40,41].

This form of the massive gravity was further generalized in [14] where the dynamical gravity was coupled to the general reference metric. Then it was a small step to the generalization of the given construction to the bimetric gravity when the fixed reference metric becomes dynamical with its own Einstein-Hilbert action [15]. It was also argued there and in [12] that this theory is ghost free. However, this analysis was discussed in [34] where it was argued that the analysis per-

\footnotetext{
${ }^{1}$ Bimetric theories of gravity were studied intensively in the past; see for example [44-46].
}

\footnotetext{
a e-mail: klu@physics.muni.cz
}

formed in [12] does not show the existence of the additional constraint in the case of the bimetric gravity. ${ }^{2}$

The non-linear massive gravity and bimetric gravity that are claimed to be ghost free are based on the specific form of the potential that contains the square root of $\hat{g}^{\mu \nu} \hat{f}_{v \rho}$. This is a rather awkward structure, which makes very difficult to find an extra constraint that could eliminate the BoulwareDeser ghost. However, as was shown in a beautiful paper [8] the square root structure suggests that the vierbein variables $E_{\mu}{ }^{A}$ could be the appropriate ones for the formulation of consistent bimetric theories. In more detail, completely new multimetric interacting spin-2 theories were proposed in [8] using the powerful vierbein formulation of general relativity and corresponding mass terms. It was argued there that due to the specific form of the interaction terms the action is linear in the lapses and shifts, which implies the existence of additional constraints that could eliminate the non-physical modes. However, we suggest that the Hamiltonian analysis presented in the given paper was not complete. In particular, the constraints corresponding to the diagonal diffeomorphism were not identified and it was not shown that they are first class constraints.

The goal of this paper is to fill this gap and perform the Hamiltonian analysis of the bimetric gravity in vierbein formulation with the simplest form of the potential between two vierbeins $E_{A}^{\mu}$ and $F_{\mu}{ }^{A}$. We explicitly show that it is crucial to analyze the time developments of the constraints corresponding to breaking the spatial rotation. It is also important to stress that when we use the parametrization of the vierbein as in [8] we should interpret $p_{a}$-which will be defined below - as a dynamical variable with no time dependence in the action. As a result the conjugate momentum vanishes and is the primary constraint of the theory. Then the

\footnotetext{
2 The Hamiltonian analysis of bimetric gravity was also performed in [35-38].
} 
requirement of the preservation of the given constraint leads to another secondary constraint that is important for further analysis.

A very important point is to identify the constraints that could be generators of the diagonal diffeomorphism. To do this we follow [33] where we introduce new variables that are functions of $N, N^{i}$ and $M, M^{i}$, which are the lapses and the shifts in $\hat{g}_{\mu \nu}$ and $\hat{f}_{\mu \nu}$, respectively. Then we determine eight new secondary constraints where four of them could correspond to the generators of the diagonal diffeomorphism on the condition that the Poisson brackets between the new Hamiltonian constraint $\overline{\mathcal{R}}$ are closed on the constraints surface, and when we add an appropriate combination of the second class constraints in order to Poisson commute with all set of constraints. A similar analysis was performed previously in the case of bimetric gravity formulated with metric variables in $[35,36]$. It turns out that in the case of bimetric theory in the vierbein formulation the situation is more complicated and we have to take into account the presence of the new secondary constraints. Then we are able to show that the Poisson bracket between the Hamiltonian constraints vanish on the constraint surface. On the other hand one can ask the question why we should use the variables introduced in [33] in the case of bimetric gravity formulated with metric variables in the case of the bimetric gravity formulated using the vierbein formalism. The answer is that using this formalism we can easily see the analogy between bimetric theories formulated using either metric of vierbein variables.

With the help of this result we proceed to the analysis of the consistency of all constraints during the time development of the system. Now due to the very remarkable structure of the vierbein formulation of bigravity we find the existence of an additional constraint, which leads to the elimination of the scalar mode in the same way as in the case of nonlinear massive gravity $[9,12]$. This result confirms the results derived in [47]. More precisely, in [47] a canonical analysis of bimetric gravity formulated in the vierbein formalism where the spin connection is treated as an independent field was performed with elegant formulations of the secondary constraints that are responsible for the elimination of the ghosts. On the contrary, our analysis is more closely related to the formulation introduced in [8], where the spin connection is not considered as an independent field, however, the constraints responsible for the elimination of ghost are much more complicated.

This paper is organized as follows. In Sect. (2) we introduce the bimetric gravity in the vierbein formalism and find its Hamiltonian, identify all constraints, and determine their constraint structure. In Sect. (3) we outline our results. Finally in the appendix we review the Hamiltonian formulation of the general relativity action formulated in the vierbein formalism.

\section{Vierbein formulation of bimetric gravity}

The general vierbein can by written in the upper triangular form and we denote this vierbein with a hat:

$\hat{E}_{\mu}^{A}=\left(\begin{array}{ll}N & N^{i} e_{i}^{a} \\ 0 & e_{i}^{a}\end{array}\right), \quad \hat{E}_{A}^{\mu}=\left(\begin{array}{ll}\frac{1}{N} & 0 \\ -\frac{N^{i}}{N} & e^{i}{ }_{a}\end{array}\right)$

where $N$ and $N^{i}$ are the four time-like components. The spatial vielbeins $e_{i}^{a}$ contain nine components, which are related to the spatial part of the metric by

$g_{i j}=e_{i}^{a} e_{j}^{b} \delta_{a b}$

Now by writing out the metric of this vierbein we find

$$
\begin{aligned}
\hat{g}_{\mu \nu} & =E_{\mu}^{A} E_{\nu}{ }^{B} \eta_{A B} \\
& =\left(\begin{array}{cc}
-N^{2}+N^{i} N_{i} & N_{i} \\
N_{j} & g_{i j}
\end{array}\right), \eta_{A B}=\operatorname{diag}(-1,1,1,1),
\end{aligned}
$$

which means that $N$ and $N^{i}$ are the usual lapse and shifts that appear in the ADM decomposition of the metric [2527]. Note that the inverse metric has the form

$\hat{g}^{\mu \nu}=\hat{E}_{A}^{\mu} \hat{E}_{B}^{v} \eta^{A B}$.

Then by definition ${ }^{3}$

$$
\begin{gathered}
\hat{E}_{\mu}^{A} \hat{E}_{A}^{v}=\delta_{\mu}^{v}, \hat{E}_{\mu}{ }^{A} \hat{E}_{B}^{\mu}=\delta_{B}^{A}, \\
e_{i}{ }^{a} e^{j}{ }_{a}=\delta_{i}{ }^{j}, e_{i}{ }^{a} e^{i}{ }_{b}=\delta_{b}^{a} .
\end{gathered}
$$

The upper triangular form does not fix the local Lorentz invariance since it leaves a residual spatial rotation. There are four components in $N, N^{i}$ and nine in the spatial vielbein. The remaining three components of the vielbein have been fixed by using the upper triangular gauge choice. It is possible to formulate an arbitrary vierbein as the action of the same boost on the upper triangular vierbein. Note that for the 3-dimensional vector $p_{a}$ we define a standard Lorentz boost as

$\Lambda(p)_{B}^{A}=\left(\begin{array}{ll}\gamma & p_{b} \\ p^{a} & \delta_{b}^{a}+\frac{1}{\gamma+1} p^{a} p_{b}\end{array}\right), \gamma=\sqrt{1+p_{a} p^{a}}$

where $p^{a}=\delta^{a b} p_{b}$ and where by definition

$\eta_{A B} \Lambda_{C}^{A} \Lambda_{D}^{B}=\eta_{C D}$,

so that

$\left(\Lambda^{-1}\right)_{B}^{A}=\left(\begin{array}{cc}\gamma & -p^{b} \\ -p_{a} & \delta_{a}^{b}+\frac{p_{a} p^{b}}{\gamma+1}\end{array}\right)$.

\footnotetext{
${ }^{3}$ For a review of the vierbein formalism, see [32].
} 
The boost takes the 4-dimensional vector $(1,0,0,0)$ into the unit normalized 4-vector

$$
\Lambda_{B}^{A}\left(\begin{array}{l}
1 \\
0
\end{array}\right)=\left(\begin{array}{c}
\gamma \\
p^{a}
\end{array}\right) \text {. }
$$

Then we write the general vierbein as the standard boost of the upper triangular vierbein,

$$
\begin{aligned}
& E_{\mu}^{A}=\Lambda(p)_{B}^{A} \hat{E}_{\mu}^{B} \\
& =\left(\begin{array}{cc}
N+N^{i} e_{i}^{a} p_{a} & N p^{b}+N^{i} e_{i}{ }^{a}\left(\delta_{a}^{b}+\frac{1}{\gamma+1} p_{a} p^{b}\right) \\
e_{i}{ }^{b} p_{a} & e_{i}{ }^{a}\left(\delta_{a}^{b}+\frac{1}{\gamma+1} p_{a} p^{b}\right)
\end{array}\right) .
\end{aligned}
$$

We see that 16 components of the general vierbein are now parameterized by the four components of $N$ and $N^{i}$ together with nine components of the spatial vielbein $e_{i}^{a}$ and three components of $p_{a}$.

It is important that the Einstein-Hilbert action is invariant under local Lorentz transformation. As a result it is possible to partially fix the gauge and express the Einstein-Hilbert action using the upper triangular form. This fact greatly simplifies the Hamiltonian formalism of general relativity in the vierbein formalism. The detailed analysis is performed in Appendix A.

Now we are ready to proceed to the vierbein formulation of the bimetric gravity when we consider bigravity with two metrics,

$\hat{g}_{\mu \nu}=E_{\mu}{ }^{A} E_{\nu}{ }^{B} \eta_{A B}, \quad \hat{f}_{\mu \nu}=F_{\mu}{ }^{A} F_{\nu}{ }^{B} \eta_{A B}$

with Einstein-Hilbert actions for both of these metrics. Then without the interaction term the action is invariant under two separate local Lorentz transformations

$E_{\mu}^{\prime A}(x)=\Lambda_{(g) B}^{A}(x) E_{\mu}^{B}(x), \quad F_{\mu}^{\prime A}(x)=\Lambda_{(f) B}^{A}(x) F_{\mu}^{B}(x)$.

The action is also invariant under two diffeomorphisms,

$$
E_{\mu}^{\prime}{ }^{A}\left(x^{\prime}\right) d f_{(1)}^{\mu}=E_{\nu}{ }^{A}(x) d x^{\nu}, \quad F_{\mu}^{\prime A}\left(x^{\prime}\right) d f_{(2)}^{\mu}=F_{\nu}{ }^{A}(x) d x^{\nu} .
$$

Then we consider the action in the form [8]

$$
\begin{aligned}
S= & \frac{M_{g}^{2}}{2} \int d^{4} x(\operatorname{det} E) R[E]+\frac{M_{f}^{2}}{2} \int d^{4} x(\operatorname{det} F) R[F] \\
& -\mu^{2} \int d^{4} x \sum_{n=0}^{4} \beta_{n}(\operatorname{det} E) S_{n}\left(E^{-1} F\right),
\end{aligned}
$$

where $\mu^{2}=\frac{1}{8} m^{2} M_{f g}^{2}$ and where $S_{n}$ are symmetric polynomials whose explicit definitions can be found in [8]. It was shown there that they can be written in terms of traces of the matrix $\mathbb{M}$ as

$$
\begin{aligned}
& S_{0}(\mathbb{M})= \\
& S_{1}(\mathbb{M})= {[\mathbb{M}] } \\
& S_{2}(\mathbb{M})= \frac{1}{2 !}\left([\mathbb{M}]^{2}-\left[\mathbb{M}^{2}\right]\right), \\
& S_{3}(\mathbb{M})= \frac{1}{3 !}\left([\mathbb{M}]^{3}-3[\mathbb{M}]\left[\mathbb{M}^{2}\right]+2\left[\mathbb{M}^{3}\right]\right), \\
& S_{4}(\mathbb{M})= \frac{1}{4 !}\left([\mathbb{M}]^{4}-6\left[\mathbb{M}^{2}\right][\mathbb{M}]^{2}+8[\mathbb{M}]\left[\mathbb{M}^{3}\right]\right. \\
&\left.+3\left[\mathbb{M}^{2}\right]^{2}-6\left[\mathbb{M}^{4}\right]\right),
\end{aligned}
$$

where $[\mathbb{M}]$ means the trace of the matrix $\mathbb{M}$. In what follows we restrict ourselves to the simplest non-trivial case corresponding to $\beta_{0}=\beta_{2}=\beta_{3}=\beta_{4}=0, \beta_{1}=1$, which, however, captures the main property of the given theory.

Now we proceed to the Hamiltonian analysis of the bimetric theory in the vierbein formulation. We use the parametrization of the general vierbein introduced in (10). Explicitly

$E_{\mu}^{A}=\Lambda(p)_{B}^{A} \hat{E}_{\mu}^{B}, \quad F_{\mu}^{A}=\Lambda(l)_{B}^{A} \hat{F}_{\mu}{ }^{B}$,

where

$$
\begin{aligned}
\hat{E}_{\mu}^{A}=\left(\begin{array}{cc}
N & N^{i} e_{i}{ }^{a} \\
0 & e_{i}^{a}
\end{array}\right), & \hat{F}_{\mu}{ }^{A}=\left(\begin{array}{cc}
M & M^{i} f_{i}^{a} \\
0 & f_{i}^{a}
\end{array}\right), \\
\hat{E}_{A}^{\mu}=\left(\begin{array}{cc}
\frac{1}{N} & 0 \\
-\frac{N^{i}}{N} & e^{i}{ }_{a}
\end{array}\right), & \hat{F}_{A}^{\mu}=\left(\begin{array}{cc}
\frac{1}{M} & 0 \\
-\frac{M^{i}}{M} & f_{a}^{i}
\end{array}\right),
\end{aligned}
$$

where $g_{i j}=e_{i}^{a} e_{j}^{b} \delta_{a b}, f_{i j}=f_{i}^{a} f_{j}^{b} \delta_{a b}$.

To proceed we use the fact that bigravity is invariant under a diagonal local Lorentz transformation, which implies that we can partially gauge fix this gauge by imposing $l_{a}=0$ [8]. Note also that since Einstein-Hilbert actions are invariant under local transformations the action depends on $p_{a}$ through the potential term only. Explicitly we find

$$
\begin{aligned}
S_{1}\left(E^{-1} F\right)= & \operatorname{Tr}\left(E^{-1} \hat{F}\right) \\
= & \frac{M}{N} \gamma+\frac{1}{N}\left(M^{i} f_{i}^{b} p_{b}-N^{i} f_{i}^{b} p_{b}\right)+e_{a}^{i} f_{i}^{a} \\
& +\frac{1}{\gamma+1}\left(e^{j}{ }_{a}^{a} p^{a}\right)\left(f_{j}^{b} p_{b}\right) .
\end{aligned}
$$

Using the Hamiltonian analysis performed in the appendix we find the following Hamiltonian:

$$
\begin{aligned}
H= & \int d^{3} \mathbf{x}\left(N \mathcal{R}_{0}^{(g)}+M \mathcal{R}_{0}^{(f)}+N^{i} \mathcal{R}_{i}^{(g)}+M^{i} \mathcal{R}_{i}^{(f)}\right. \\
& \left.+\mu^{2} N e \mathcal{V}+\Lambda_{(g)}^{a b} L_{a b}^{(g)}+\Lambda_{(f)}^{a b} L_{a b}^{(f)}\right)
\end{aligned}
$$


where

$$
\begin{aligned}
\mathcal{R}_{0}^{(g)}= & \frac{1}{M_{g}^{2} \sqrt{g}} \pi^{i j} \mathcal{G}_{i j k l} \pi^{k l}-M_{g}^{2} \sqrt{g} R^{(g)}, \\
\mathcal{R}_{0}^{(f)}= & \frac{1}{M_{f}^{2} \sqrt{f}} \rho^{i j} \tilde{\mathcal{G}}_{i j k l} \rho^{k l}-M_{f}^{2} \sqrt{f} R^{(f)}, \\
\mathcal{R}_{i}^{(g)}= & -2 g_{i j} \nabla_{k} \pi^{k j}, \quad L_{a b}^{(g)}=e_{i a} \pi_{b}^{i}-e_{i b} \pi_{a}^{i}, \\
\mathcal{R}_{i}^{(f)}= & -2 f_{i j} \tilde{\nabla}_{k} \rho^{k j}, \quad L_{a b}^{(f)}=f_{i a} \rho_{b}^{i}-f_{i b} \rho_{a}^{i}, \\
\mathcal{V}= & \frac{M}{N} \gamma+\frac{1}{N}\left(M^{i} f_{i}^{b} p_{b}-N^{i} f_{i}^{b} p_{b}\right) \\
& +e_{a}^{i} f_{i}{ }^{a}+\frac{1}{\gamma+1}\left(e^{j}{ }_{a} p^{a}\right)\left(f_{j}^{b} p_{b}\right),
\end{aligned}
$$

and where $\pi^{i j}$ and $\rho^{i j}$ are the momenta conjugate to $g_{i j}$ and $f_{i j}$, respectively. Further $\nabla_{i}$ and $\tilde{\nabla}_{i}$ are covariant derivatives evaluated using the metric components $g_{i j}$ and $f_{i j}$, respectively. Finally note that $\mathcal{G}^{i j k l}$ and $\tilde{\mathcal{G}}^{i j k l}$ are de Witt metrics defined as

$$
\begin{aligned}
& \mathcal{G}^{i j k l}=\frac{1}{2}\left(g^{i k} g^{j l}+g^{i l} g^{j k}\right)-g^{i j} g^{k l}, \\
& \tilde{\mathcal{G}}^{i j k l}=\frac{1}{2}\left(f^{i k} f^{j l}+f^{i l} f^{j k}\right)-f^{i j} f^{k l},
\end{aligned}
$$

with inverse

$\begin{aligned} \mathcal{G}_{i j k l} & =\frac{1}{2}\left(g_{i k} g_{j l}+g_{i l} g_{j k}\right)-\frac{1}{2} g_{i j} g_{k l}, \\ \tilde{\mathcal{G}}_{i j k l} & =\frac{1}{2}\left(f_{i k} f_{j l}+f_{i l} f_{j k}\right)-\frac{1}{2} f_{i j} f_{k l},\end{aligned}$

which obey the relations

$\mathcal{G}_{i j k l} \mathcal{G}^{k l m n}=\frac{1}{2}\left(\delta_{i}^{m} \delta_{j}^{n}+\delta_{i}^{n} \delta_{j}^{m}\right)$,

$\tilde{\mathcal{G}}_{i j k l} \tilde{\mathcal{G}}^{k l m n}=\frac{1}{2}\left(\delta_{i}^{m} \delta_{j}^{n}+\delta_{i}^{n} \delta_{j}^{m}\right)$.

Also note that $e \equiv \operatorname{det} e$. We have also included the primary constraints $L_{a b}^{(g)} \approx 0, L_{a b}^{(f)} \approx 0$ into the definition of the Hamiltonian.

An important point is to identify four constraints with the property that their Poisson brackets vanish on the constraint surface and hence could be part of the generators of the diagonal diffeomorphism. In order to do this we proceed as in [33] and introduce the following variables:

$\bar{N}=\sqrt{N M}, n=\sqrt{\frac{N}{M}}, \bar{N}^{i}=\frac{1}{2}\left(N^{i}+M^{i}\right), n^{i}=\frac{N^{i}-M^{i}}{\sqrt{N M}}$,

$N=\bar{N} n, M=\frac{\bar{N}}{n}, M^{i}=\bar{N}^{i}-\frac{1}{2} n^{i} \bar{N}, N^{i}=\bar{N}^{i}+\frac{1}{2} n^{i} \bar{N}$.
Note that their conjugate momenta are the primary constraints of the theory

$\bar{P} \approx 0, \quad p \approx 0, \quad P_{i} \approx 0, \quad p_{i} \approx 0$

with the following non-zero Poisson brackets:

$$
\begin{array}{rlrl}
\{\bar{N}(\mathbf{x}), \bar{P}(\mathbf{y})\} & =\delta(\mathbf{x}-\mathbf{y}), & & \{n(\mathbf{x}), p(\mathbf{y})\}=\delta(\mathbf{x}-\mathbf{y}), \\
\left\{\bar{N}^{i}(\mathbf{x}), P_{j}(\mathbf{y})\right\} & =\delta_{j}^{i} \delta(\mathbf{x}-\mathbf{y}), & \left\{n^{i}(\mathbf{x}), p_{j}(\mathbf{y})\right\}=\delta_{j}^{i} \delta(\mathbf{x}-\mathbf{y}) .
\end{array}
$$

It is also important to stress that the absence of the time derivative of $p_{a}$ in the action implies the following primary constraint:

$k^{a} \approx 0$,

where $k^{a}$ is the momentum conjugate to $p_{a}$ with non-zero Poisson bracket,

$\left\{p_{a}(\mathbf{x}), k^{b}(\mathbf{y})\right\}=\delta_{a}^{b} \delta(\mathbf{x}-\mathbf{y})$.

We also have to identify the constraints that are generators of the diagonal spatial rotations of the vielbeins $e_{i}{ }^{a}, f_{i}{ }^{a}$. These constraints are given as linear combinations of $L_{a b}^{(g)}, L_{a b}^{(f)}$ and $k^{a}$. Explicitly, we introduce the following set of constraints:

$L_{a b}^{\mathrm{diag}} \approx 0, \quad L_{a b}^{\mathrm{br}} \approx 0, \quad k^{a} \approx 0$,

where

$$
\begin{aligned}
L_{a b}^{\mathrm{diag}} & =e_{i a} \pi_{b}^{i}-e_{i b} \pi_{a}^{i}+f_{i a} \rho_{b}^{i}-f_{i b} \rho_{a}^{i}+p_{a} k_{b}-p_{b} k_{a}, \\
L_{a b}^{\mathrm{br}} & =e_{i a} \pi_{b}^{i}-e_{i b} \pi_{a}^{i}-f_{i a} \rho_{b}^{i}+f_{i b} \rho_{a}^{i}-p_{a} k_{b}+p_{b} k_{a},
\end{aligned}
$$

where $\pi_{a}^{i}, \rho_{a}^{i}$ are momenta conjugate to $e_{i}^{a}, f_{i}{ }^{a}$, respectively. Collecting all these terms we find the following form of the Hamiltonian:

$$
\begin{aligned}
H= & \int d^{3} \mathbf{x}\left(\bar{N} \overline{\mathcal{R}}+\bar{N}^{i} \overline{\mathcal{R}}_{i}+\Lambda_{\mathrm{diag}}^{a b} L_{a b}\right. \\
& \left.+\Lambda_{\mathrm{br}}^{a b} L_{a b}^{\mathrm{br}}+v_{a} k^{a}+v_{n} p+v^{i} p_{i}+V_{\bar{N}} \bar{P}+V^{i} P_{i}\right),
\end{aligned}
$$

where

$$
\begin{aligned}
\overline{\mathcal{R}}= & n \mathcal{R}_{0}^{(g)}+\frac{1}{n} \mathcal{R}_{0}^{(f)}+\frac{1}{2} n^{i} \mathcal{R}_{i}^{(g)}-\frac{1}{2} n^{i} \mathcal{R}_{i}^{(f)} \\
& +\mu^{2} e \tilde{\mathcal{V}}, \quad \overline{\mathcal{R}}_{i}=\mathcal{R}_{i}^{(g)}+\mathcal{R}_{i}^{(f)}
\end{aligned}
$$

where

$$
\tilde{\mathcal{V}}=\frac{\gamma}{n}-n^{i} f_{i}^{a} p_{a}+n e^{i}{ }_{a} f_{i}^{a}+\frac{n}{\gamma+1}\left(e^{i}{ }_{a} p^{a}\right)\left(f_{i}^{b} p_{b}\right) .
$$


Now we proceed to the analysis of the time development of the primary constraints (25) and (27),

$$
\begin{aligned}
& \partial_{t} \bar{P}=\{\bar{P}, H\}=-\overline{\mathcal{R}} \approx 0, \\
& \partial_{t} P_{i}=\left\{P_{i}, H\right\}=-\overline{\mathcal{R}}_{i} \approx 0, \partial_{t} p=\{p, H\}=-\mathcal{R}_{0}^{(g)}+\frac{1}{n^{2}} \mathcal{R}_{0}^{(f)} \\
&-\mu^{2} e \frac{\delta \tilde{\mathcal{V}}}{\delta n} \equiv \mathcal{G}_{n} \approx 0, \\
& \partial_{t} p_{i}=\left\{p_{i}, H\right\}=-\frac{1}{2}\left(\mathcal{R}_{i}^{(g)}-\mathcal{R}_{i}^{(f)}\right) \\
&-\mu^{2} e \frac{\delta \tilde{\mathcal{V}}}{\delta n^{i}} \equiv \mathcal{S}_{i} \approx 0, \\
& \partial_{t} k^{a}=\left\{k^{a}, H\right\}=-\mu^{2} e \frac{\delta \tilde{\mathcal{V}}}{\delta p_{a}} \equiv \mathcal{K}^{a} \approx 0 .
\end{aligned}
$$

Finally we have to check the preservation of the constraints $L_{a b}^{\text {diag }} \approx 0, L_{a b}^{\mathrm{br}} \approx 0$. Firstly due to the fact that $\mathcal{R}_{0}^{(g)}, \mathcal{R}_{0}^{(f)}, \mathcal{R}_{i}^{(g)}, \mathcal{R}_{i}^{(f)}$ have vanishing Poisson brackets with $L_{a b}^{(g)}, L_{a b}^{(f)}$ according to (118) we find that they have also a vanishing Poisson brackets with both $L_{a b}^{\mathrm{diag}}$ and $L_{a b}^{\mathrm{br}}$. Then the non-zero contribution could follow from the Poisson bracket between $L_{a b}^{\mathrm{diag}}, L_{a b}^{\mathrm{br}}$ and $\tilde{\mathcal{V}}$. Now with the help of the following Poisson brackets:

$$
\begin{aligned}
& \left\{L_{a b}^{\mathrm{diag}}(\mathbf{x}), e_{i}^{c}(\mathbf{y})\right\}=\left(e_{i b} \delta_{a}^{c}-e_{i a} \delta_{b}^{c}\right)(\mathbf{x}) \delta(\mathbf{x}-\mathbf{y}), \\
& \left\{L_{a b}^{\mathrm{diag}}(\mathbf{x}), e_{c}^{i}(\mathbf{y})\right\}=\left(\delta_{a c} e_{b}^{i}-\delta_{b c} e_{a}^{i}\right)(\mathbf{x}) \delta(\mathbf{x}-\mathbf{y}), \\
& \left\{L_{a b}^{\mathrm{diag}}(\mathbf{x}), f_{i}^{c}(\mathbf{y})\right\}=\left(f_{i b} \delta_{a}^{c}-f_{i a} \delta_{b}^{c}\right)(\mathbf{x}) \delta(\mathbf{x}-\mathbf{y}), \\
& \left\{L_{a b}^{\mathrm{diag}}(\mathbf{x}), f_{c}^{i}(\mathbf{y})\right\}=\left(\delta_{a c} f_{b}^{i}-\delta_{b c} f_{a}^{i}\right)(\mathbf{x}) \delta(\mathbf{x}-\mathbf{y}), \\
& \left\{L_{a b}^{\mathrm{diag}}(\mathbf{x}), p_{c}(\mathbf{y})\right\}=-\left(p_{a} \delta_{b c}-p_{b} \delta_{a c}\right)(\mathbf{x}) \delta(\mathbf{x}-\mathbf{y}), \\
& \left\{L_{a b}^{\mathrm{diag}}(\mathbf{x}), p_{c} p^{c}(\mathbf{y})\right\}=0,
\end{aligned}
$$

and also

$$
\begin{aligned}
& \left\{L_{a b}^{\mathrm{br}}(\mathbf{x}), e_{i}^{c}(\mathbf{y})\right\}=\left(e_{i b} \delta_{a}^{c}-e_{i a} \delta_{b}^{c}\right)(\mathbf{x}) \delta(\mathbf{x}-\mathbf{y}), \\
& \left\{L_{a b}^{\mathrm{br}}(\mathbf{x}), e_{c}^{i}(\mathbf{y})\right\}=\left(\delta_{a c} e_{b}^{i}-\delta_{b c} e_{a}^{i}\right)(\mathbf{x}) \delta(\mathbf{x}-\mathbf{y}), \\
& \left\{L_{a b}^{\mathrm{br}}(\mathbf{x}), f_{i}^{c}(\mathbf{y})\right\}=-\left(f_{i b} \delta_{a}^{c}-f_{i a} \delta_{b}^{c}\right)(\mathbf{x}) \delta(\mathbf{x}-\mathbf{y}), \\
& \left\{L_{a b}^{\mathrm{br}}(\mathbf{x}), f_{c}^{i}(\mathbf{y})\right\}=-\left(\delta_{a c} f_{b}^{i}-\delta_{b c} f_{a}^{i}\right)(\mathbf{x}) \delta(\mathbf{x}-\mathbf{y}), \\
& \left\{L_{a b}^{\mathrm{br}}(\mathbf{x}), p_{c}(\mathbf{y})\right\}=\left(p_{a} \delta_{b c}-p_{b} \delta_{a c}\right)(\mathbf{x}) \delta(\mathbf{x}-\mathbf{y}), \\
& \left\{L_{a b}^{\mathrm{br}}(\mathbf{x}), p_{c} p^{c}(\mathbf{y})\right\}=0,
\end{aligned}
$$

we find that the constraint $L_{a b}^{\text {diag }} \approx 0$ is preserved during the time evolution of the system, while the requirement of the preservation of the constraint $L_{a b}^{\mathrm{br}}$ implies

$$
\begin{aligned}
\partial_{t} L_{a b}^{\mathrm{br}}(\mathbf{x})= & \left\{L_{a b}^{\mathrm{br}}(\mathbf{x}), H\right\} \\
= & 2 \mu^{2} \bar{N} e n\left[\left(e_{b}^{j} f_{j a}-e_{a}^{j} f_{j b}\right)\right. \\
& +\frac{1}{\gamma+1}\left[\left(p_{a} e_{b}^{j}-p_{b} e_{a}^{j}\right) f_{j}^{d} p_{d}\right] \\
\equiv & 2 \mu^{2} e \bar{N} n \mathcal{T}_{a b} \approx 0,
\end{aligned}
$$

where we introduced new secondary constraints $\mathcal{T}_{a b}=-\mathcal{T}_{b a}$

$\mathcal{T}_{a b}=e^{j} f_{j a}-e^{j} f_{j b}+\frac{1}{\gamma+1}\left(p_{a} e_{b}^{j}-p_{b} e_{a}^{j}\right) f_{j}^{d} p_{d}$.

As we will see below the existence of these constraints will be crucial for the consistency of the theory.

2.1 Calculation of the Poisson brackets between $\overline{\mathcal{R}}, \overline{\mathcal{R}}_{i}$

Before we proceed to the analysis of the stability of all constraints we would like to show that the Poisson brackets between the constraints $\overline{\mathcal{R}}$ and $\overline{\mathcal{R}}_{i}$ vanish on the constraint surface spanned by all constraints. To begin with we introduce the smeared form of the constraint $\overline{\mathcal{R}}$,

$\mathbf{T}(N)=\int d^{3} \mathbf{x} N \overline{\mathcal{R}}$.

Then using the Poisson brackets given in (118) and the following similar analysis as in the case of metric formulations of bigravity we obtain $[35,36]$

$$
\begin{aligned}
\left\{\mathbf{T}_{T}(N), \mathbf{T}_{T}(M)\right\}= & \frac{1}{2} \mathbf{T}_{S}\left(\left(N \partial_{i} M-M \partial_{i} N\right) n^{2} g^{i j}\right) \\
& +\frac{1}{2} \mathbf{T}_{S}\left(\left(N \partial_{i} M-M \partial_{i} N\right) \frac{1}{n^{2}} f^{i j}\right) \\
& +\frac{1}{4} \mathbf{T}_{S}\left(\left(N \partial_{i} M-M \partial_{i} N\right) n^{i} n^{j}\right) \\
& -\mathbf{G}_{S}\left(\left(N \partial_{i} M-M \partial_{i} N\right) n^{2} g^{i j}\right) \\
& +\mathbf{G}_{S}\left(\left(N \partial_{i} M-M \partial_{i} N\right) \frac{1}{n^{2}} f^{i j}\right) \\
& -\frac{1}{2} \mathbf{G}_{T}\left(\left(N \partial_{i} M-M \partial_{i} N\right) n^{i} n\right) \\
& +\int d^{3} \mathbf{x}\left(N \partial_{i} M-M \partial_{i} N\right) \Sigma^{i},
\end{aligned}
$$

where

$\mathbf{G}_{T}(N)=\int d^{3} \mathbf{x} N \mathcal{G}_{n}, \quad \mathbf{G}_{S}\left(N^{i}\right)=\int d^{3} \mathbf{x} N^{i} \mathcal{S}_{i}$,

and where

$$
\begin{aligned}
\Sigma^{i}[\tilde{\mathcal{V}}]= & \gamma \frac{n^{i}}{n}-n e_{a}^{i} f_{j}{ }^{a} n^{j}-\frac{n}{\gamma+1} e_{b}^{i} p^{b} n^{j} f_{j}^{a} p_{a} \\
& +n^{2} g^{i j} f_{j}^{a} p_{a}-\frac{1}{n^{2}} f_{a}^{i} p^{a} .
\end{aligned}
$$


Note also that we used the extended version of the constraint $\overline{\mathcal{R}}_{i}$ given in (47) and we omitted terms proportional to $L_{a b}^{(g)}, L_{a b}^{(f)}$ given in (118).

Our goal is to show that $\Sigma^{i}[\mathcal{V}]$ vanishes on the constraint surface. To do this we use the fact that $n^{i}$ can be expressed from the constraint $\mathcal{K}^{a}$

$$
\begin{aligned}
n^{i}= & f_{a}^{i}\left(\frac{1}{\gamma n} p^{a}-n\left(e_{b}^{j} p^{b}\right)\left(f_{j}^{c} p_{c}\right) \frac{1}{(1+\gamma)^{2} \gamma} p^{a}\right. \\
& \left.+\frac{1}{1+\gamma}\left(f_{j}^{a} e_{b}^{j} p^{b}+f_{j}^{b} p_{b} e^{j a}\right)+H_{a} \mathcal{K}^{a}\right),
\end{aligned}
$$

where $H_{a}$ are functions that depend on the phase space variables whose explicit form is not important for us. To proceed we use the fact that from the constraint $\mathcal{T}_{a b}$ we derive

$$
\begin{aligned}
f_{a}^{i} f_{j}^{b} p_{b} e^{j a}= & \frac{1}{\gamma}\left(e^{i b} p_{b}+\frac{1}{1+\gamma} f^{i a} p_{a}\left(f_{k}{ }^{d} p_{d}\right)\left(e^{k}{ }_{b} p^{b}\right)\right) \\
& -\frac{f_{a}^{i}}{2 \gamma \sqrt{e} \sqrt{f}} \mathcal{G}^{a b} p_{b} .
\end{aligned}
$$

Inserting this expression into (43) we find

$n^{i}=\frac{1}{\gamma n} f_{a}^{i} p^{a}+\frac{n}{\gamma} e^{i b} p_{b}$

up to terms proportional to $\mathcal{T}_{a b}$ and $\mathcal{K}^{a}$. Finally inserting this result into (42) and after some calculations we find the desired result,

$$
\Sigma^{i}[\tilde{\mathcal{V}}]=F_{a} \mathcal{K}^{a}+G^{a b} \mathcal{T}_{a b} \approx 0
$$

Then collecting (40) together with (46) we find that the Poisson bracket between $\overline{\mathcal{R}}$ is proportional to the constraints $\overline{\mathcal{R}}_{i}, \mathcal{G}_{n}, \mathcal{S}_{i}, \mathcal{K}^{a}, \mathcal{T}_{a b}$, which means that it vanishes on the constraint surface. This is a very important result. Note also the importance of the constraints $\mathcal{K}^{a}, \mathcal{T}_{a b}$ for the closure of the Poisson brackets between $\overline{\mathcal{R}}$.

As the next step we calculate the Poisson brackets with the constraints $\overline{\mathcal{R}}_{i}$. However, it turns out that it is more convenient to consider the following extension:

$$
\begin{aligned}
\overline{\mathcal{R}}_{i}= & \partial_{i} n p+\partial_{i} n^{j} p_{j}+\partial_{j}\left(n^{j} p_{i}\right)+\partial_{i} p_{a} k^{a} \\
& +\mathcal{R}_{i}^{(e)}+\frac{1}{2} \omega_{i}^{a b}(e) L_{a b}^{(e)}+\mathcal{R}_{i}^{(f)}+\frac{1}{2} \omega_{i}^{a b}(f) L_{a b}^{(f)} .
\end{aligned}
$$

Let us define its smeared form,

$$
\mathbf{T}_{S}\left(N^{i}\right)=\int d^{3} \mathbf{x} N^{i} \overline{\mathcal{R}}_{i}
$$

Then we find the following Poisson brackets:

$$
\begin{aligned}
& \left\{\mathbf{T}_{S}\left(N^{i}\right), e_{i}{ }^{c}\right\}=-\partial_{i} N^{j} e_{j}^{c}-N^{j} \partial_{j} e_{i}^{c}, \\
& \left\{\mathbf{T}_{S}\left(N^{i}\right), e^{i}{ }_{c}\right\}=\partial_{j} N^{i} e_{c}^{j}-N^{j} \partial_{j} e_{c}^{i}, \\
& \left\{\mathbf{T}_{S}\left(N^{i}\right), f_{c}^{i}\right\}=\partial_{j} N^{i} f_{c}^{j}-N^{j} \partial_{j} f_{c}^{i}, \\
& \left\{\mathbf{T}_{S}\left(N^{i}\right), f_{i}^{c}\right\}=-\partial_{i} N^{j} f_{j}^{c}-N^{j} \partial_{j} f_{i}^{c}, \\
& \left\{\mathbf{T}_{S}\left(N^{i}\right), n^{i}\right\}=-N^{j} \partial_{j} n^{i}+\partial_{j} N^{i} n^{j}, \\
& \left\{\mathbf{T}_{S}\left(N^{i}\right), p_{a}\right\}=-N^{i} \partial_{i} p_{a}, \\
& \left\{\mathbf{T}_{S}\left(N^{i}\right), n\right\}=-N^{i} \partial_{i} n,
\end{aligned}
$$

which shows that $\mathbf{T}_{S}\left(N^{i}\right)$ is the generator of the diagonal spatial diffeomorphism.

Now we are ready to proceed to the calculation of the Poisson bracket between $\mathbf{T}_{S}\left(N^{i}\right)$ defined in (48) and $\mathbf{T}_{T}(N)$. In fact, using (49) we easily find

$$
\left\{\mathbf{T}_{S}\left(N^{i}\right), e \tilde{\mathcal{V}}\right\}=-N^{k} \partial_{k}[e \tilde{\mathcal{V}}]-\partial_{k} N^{k} e \tilde{\mathcal{V}}
$$

Finally, we should calculate the Poisson bracket between $\mathbf{T}_{S}\left(N^{i}\right)$ and $\mathcal{R}_{i}^{(f)}, \mathcal{R}_{i}^{(g)}$ and $\mathcal{R}_{0}^{(g)}, \mathcal{R}_{0}^{(f)}$. This is really an easy task on using the results given in (118) so that we find

$$
\left\{\mathbf{T}_{S}\left(N^{i}\right), \mathbf{T}_{T}(N)\right\}=\mathbf{T}_{T}\left(N^{i} \partial_{i} M\right)
$$

up to the terms proportional to the primary constraints $L_{a b}^{(g)} \approx$ $0, L_{a b}^{(f)} \approx 0$. In the same way we can find that

$$
\left\{\mathbf{T}_{S}\left(N^{i}\right), \mathbf{T}_{S}\left(M^{j}\right)\right\}=\mathbf{T}_{S}\left(\left(N^{i} \partial_{i} M^{j}-M^{j} \partial_{i} N^{j}\right)\right) .
$$

Using these results we are ready to proceed to the analysis of the stability of constraints.

\subsection{Analysis of stability of constraints}

In this section we perform the analysis of the stability of all constraints. Note that for the potential $\tilde{\mathcal{V}}$ given in (33) the constraints $\mathcal{G}_{n}, \mathcal{S}_{i}, \mathcal{K}^{a}$ have the form

$$
\begin{aligned}
\mathcal{G}_{n}= & -\mathcal{R}_{0}^{(g)}+\frac{1}{n^{2}} \mathcal{R}_{0}^{(f)} \\
& +\mu^{2} e\left(\frac{\gamma}{n^{2}}-e^{i}{ }_{a} f_{i}{ }^{a}-\frac{1}{1+\gamma}\left(e^{i}{ }_{a} p^{a}\right)\left(f_{i}^{b} p_{b}\right)\right), \\
\mathcal{S}_{i}= & -\frac{1}{2}\left(\mathcal{R}_{i}^{(g)}-\mathcal{R}_{i}^{(f)}\right)+\mu^{2} e f_{i}^{a} p_{a}, \\
\mathcal{K}^{a}= & \mu^{2} e\left(\frac{p^{a}}{\gamma n}-n^{i} f_{i}{ }^{a}-n\left(e^{j}{ }_{c} p^{c}\right)\left(f_{j}^{b} p_{b}\right) \frac{p^{a}}{(1+\gamma)^{2} \gamma}\right. \\
& \left.+\frac{n}{1+\gamma}\left(f_{j}{ }^{a} e^{j}{ }_{b} p^{b}+f_{j}{ }^{b} p_{b} e^{j a}\right)\right),
\end{aligned}
$$


$\mathcal{T}_{a b}=\left(e_{b}^{j} f_{j a}-e^{j}{ }_{a} f_{j b}\right)+\frac{1}{1+\gamma}\left(p_{a} e_{b}^{j}-p_{b} e_{a}^{j}\right) f_{j}{ }^{d} p_{d}$.

It turns out that these constraints could be simplified considerably. First of all we have the following relation:

$\overline{\mathcal{R}}+n^{i} \mathcal{S}_{i}+n \mathcal{G}_{n}=\frac{2}{n}\left(\mathcal{R}_{0}^{(f)}+\mu^{2} e \gamma\right)$,

so that we can consider as an independent constraint the following one:

$\mathcal{G}_{n}^{\prime}=\mathcal{R}_{0}^{(f)}+\mu^{2} e \gamma$

In the previous section we also found the relation

$n^{i}=\frac{1}{\gamma n} f_{a}^{i} p^{a}+\frac{n}{\gamma} e^{i b} p_{b}+H_{a} \mathcal{K}^{a}+G^{a b} \mathcal{T}_{a b}$,

so that it is possible to define a new independent constraint $\tilde{\mathcal{K}}^{i}$,

$\tilde{\mathcal{K}}^{i}=n^{i}-\frac{1}{\gamma n} f_{a}^{i} p^{a}-\frac{n}{\gamma} e^{i b} p_{b}$

Then we have the following set of independent secondary constraints: $\mathcal{G}_{n}^{\prime}, \tilde{\mathcal{K}}^{i}, \mathcal{S}_{i}, \mathcal{T}_{a b}$, so that the total Hamiltonian has the form

$$
\begin{aligned}
H_{T}= & \int d^{3} \mathbf{x}\left(\bar{N} \overline{\mathcal{R}}+\bar{N}^{i} \overline{\mathcal{R}}_{i}+\Lambda_{d i a g}^{a b} L_{a b}+V_{\bar{N}} \bar{P}+V^{i} P_{i}\right. \\
& +\Gamma_{n} \mathcal{G}_{n}^{\prime}+\Omega_{i} \tilde{\mathcal{K}}^{i}+\Gamma^{i} \mathcal{S}_{i}+\Gamma^{a b} \mathcal{T}_{a b}+v_{n} p \\
& \left.+v^{i} p_{i}+v_{a} k^{a}+v^{a b} L_{a b}^{\mathrm{br}}\right) .
\end{aligned}
$$

Now we are ready to proceed to the analysis of the stability of all constraints. We begin with the constraints $p_{i} \approx 0$,

$\partial_{t} p_{i}=\left\{p_{i}, H_{T}\right\} \approx-\mu^{2} e \Omega_{i}=0$,

which has the solution $\Omega_{i}=0$, where $\Omega_{i}$ is the Lagrange multiplier corresponding to the constraint $\tilde{\mathcal{K}}^{i}$. In the case of $p$ we find

$\partial_{t} p=\left\{p, H_{T}\right\} \approx 0$.

Further, the requirement of the preservation of $L_{a b}^{\mathrm{br}}$ takes the form

$\partial_{t} L_{a b}^{\mathrm{br}}=\left\{L_{a b}^{\mathrm{br}}, H_{T}\right\}=\Gamma^{c d} \triangle_{L_{a b}^{\mathrm{br}},} \mathcal{T}_{c d}=0$,

where $\left\{L_{a b}^{\mathrm{br}}(\mathbf{x}), \mathcal{T}_{c d}(\mathbf{y})\right\}=\triangle_{L_{a b}^{\mathrm{br}},}, \mathcal{T}_{c d}(\mathbf{x}) \delta(\mathbf{x}-\mathbf{y})$ and where we used the fact that $\Omega_{i}=0$ together with

$$
\left\{L_{a b}^{\mathrm{br}}(\mathbf{x}), \mathcal{G}_{n}^{\prime}(\mathbf{y})\right\}=\left\{L_{a b}^{\mathrm{br}}(\mathbf{x}), \mathcal{S}_{i}(\mathbf{y})\right\}=0 .
$$

Then it can be explicitly checked that the matrix $\triangle_{L_{a b}^{\mathrm{br}},}, \mathcal{T}_{c d}$ is non-singular and hence the solution of (61) is $\Gamma^{c d}=0$.

Using these results it is easy to analyze the requirement of the preservation of the constraints $k^{a} \approx 0$,

$\partial_{t} k^{a}=\left\{k^{a}, H_{T}\right\} \approx-\mu^{2} e \frac{p^{a}}{\gamma}-\Gamma^{i} \mu^{2} e f_{i}^{a}=0$,

so that we obtain

$\Gamma^{i}=-\frac{p^{a}}{\gamma} f_{a}^{i} \Gamma_{n}$

This result, however, suggests us to consider as an independent constraint the following one:

$\tilde{\mathcal{G}}_{n}=\mathcal{R}_{0}^{(f)}+\mu^{2} e \gamma-\frac{1}{\gamma} \mathcal{S}_{i} f_{a}^{i} p^{a}$

and the following total Hamiltonian:

$$
\begin{aligned}
H_{T}= & \int d^{3} \mathbf{x}\left(\bar{N} \overline{\mathcal{R}}+\bar{N}^{i} \overline{\mathcal{R}}_{i}+\Lambda_{d i a g}^{a b} L_{a b}+V_{\bar{N}} \bar{P}+V^{i} P_{i}\right. \\
& +\Gamma^{i} \mathcal{S}_{i}+\Omega_{i} \tilde{\mathcal{K}}^{i}+\Gamma_{n} \tilde{\mathcal{G}}_{n}+v^{n} p+v^{i} p_{i}+v_{a} k^{a} \\
& \left.+v^{a b} L_{a b}^{\mathrm{br}}+\Gamma^{a b} \mathcal{T}_{a b}\right) .
\end{aligned}
$$

Repeating the analysis as above we find that $p$ is trivially preserved and also $\Omega_{i}=\Gamma^{a b}=0$. Further, the time evolution of the constraint $k^{a} \approx 0$ is given by the equation

$\partial_{t} k^{a}=\left\{k^{a}, H_{T}\right\} \approx-\Gamma^{i} \mu^{2} e f_{i}^{a}=0$,

which due to the fact that $f_{i}{ }^{a}$ is non-singular implies that $\Gamma^{i}=0$. Finally it is also clear that $\bar{P}, P_{i}$ are trivially preserved.

Now we proceed to the analysis of the time evolution of the constraint $\tilde{\mathcal{G}}_{n}, \mathcal{S}_{i}, \tilde{\mathcal{K}}^{i}$ together with $\overline{\mathcal{R}}$ and $\overline{\mathcal{R}}_{i}$. First of all it is easy to see that the secondary constraints $\tilde{\mathcal{G}}_{n}, \mathcal{S}_{i}, \tilde{\mathcal{K}}^{i}, \tilde{\mathcal{G}}_{a b}$ are invariant under a diagonal spatial diffeomorphism. Then with the help of (51) and (52) we find that $\overline{\mathcal{R}}_{i}$ are preserved during the time evolution of the system.

A more interesting situation occurs in the case of the time evolution of the constraints $\tilde{\mathcal{G}}_{n}$ and $\overline{\mathcal{R}}$, which is mainly determined by the following Poisson bracket:

$$
\left\{\mathbf{T}_{T}(N), \int d^{3} \mathbf{x} M \tilde{\mathcal{G}}_{n}\right\} \approx \int d^{3} \mathbf{x} N(\mathbf{x}) M(\mathbf{x}) n(\mathbf{x}) \tilde{\mathcal{G}}_{n}^{I I}(\mathbf{x}),
$$

where the explicit form of $\tilde{\mathcal{G}}_{n}^{I I}$, which is rather complicated, is not important for us; but it is important to stress that $\tilde{\mathcal{G}}_{n}^{I I}$ does not depend on $n$ and it is proportional to $\mu^{2}$, which means that the given constraint vanishes in the decoupling 
case when $\mu^{2}=0$, which is expected. ${ }^{4}$ Note that in the course of the calculation we use the fact that we can write $\overline{\mathcal{R}}$ as

$$
\begin{aligned}
\overline{\mathcal{R}}= & n \mathcal{R}_{0}^{(g)}+\frac{1}{n} \tilde{\mathcal{G}}_{n}+\mathcal{S}_{i}\left[\frac{1}{\gamma n} f_{a}^{i} p^{a}-n^{i}\right] \\
& +\mu^{2} e n\left[e^{i}{ }_{a} f_{i}{ }^{a}+\frac{n}{1+\gamma}\left(e^{i}{ }_{a} p^{a}\right)\left(f_{i}{ }^{b} p_{b}\right)\right] \\
= & n\left[\mathcal{R}_{0}^{(g)}-\frac{1}{\gamma} e^{i b} p_{b} \mathcal{S}_{i}+\mu^{2} e\left(e^{i}{ }_{a} f_{i}{ }^{a}\right.\right. \\
& \left.\left.+\frac{1}{1+\gamma}\left(e^{i}{ }_{a} p^{a}\right)\left(f_{i}{ }^{b} p_{b}\right)\right)\right]-\mathcal{K}^{i} \mathcal{S}_{i}+\frac{1}{n} \tilde{\mathcal{G}}_{n},
\end{aligned}
$$

and we also used the fact that the Poisson bracket $\left\{\tilde{\mathcal{G}}_{n}(\mathbf{x}), \tilde{\mathcal{G}}_{n}(\mathbf{y})\right\}$ is weakly zero as follows from

$$
\begin{aligned}
& \left\{\int d^{3} \mathbf{x} N \tilde{\mathcal{G}}_{n}(\mathbf{x}), \int d^{3} \mathbf{y} M \tilde{\mathcal{G}}_{n}(\mathbf{y})\right\} \\
& =\frac{1}{2} \mathbf{T}_{S}\left(\left(N \partial_{i} M-M \partial_{i} N\right) f^{i j}\right) \\
& \quad+\frac{1}{4} \mathbf{T}_{S}\left(\frac{1}{\gamma^{2}} f_{a}^{i} p^{a} f_{b}^{j} p^{b}\left(\partial_{i} N M-M \partial_{i} N\right)\right) \\
& \quad+\int d^{3} \mathbf{x}\left(N \partial_{i} M-M \partial_{i} N\right) f^{i j} \mathcal{S}_{j} \\
& \quad-\frac{1}{2} \int d^{3} \mathbf{x}\left(N \partial_{i} M-M \partial_{i} N\right) \frac{f_{a}^{i} p^{2}}{\gamma} \mathcal{G}_{n}^{\prime} \approx 0 .
\end{aligned}
$$

However, it is a crucial and non-trivial fact that the Poisson bracket (68) does not contain terms proportional to $M \partial_{i} N$ or $M \partial_{i} N$ and it is linear in $n$. Then the local form (68) is

$$
\left\{\overline{\mathcal{R}}(\mathbf{x}), \tilde{\mathcal{G}}_{n}(\mathbf{y})\right\} \approx n(\mathbf{x}) \tilde{\mathcal{G}}_{n}^{I I}(\mathbf{x}) \delta(\mathbf{x}-\mathbf{y}),
$$

so that there are no derivatives of the delta function on the right side of the previous equation. This fact is very important for the consistency of the given theory.

Now we are ready to proceed to the analysis of the consistency of the secondary constraints. In the case of $\tilde{\mathcal{G}}_{n}$ we obtain

$\partial_{t} \tilde{\mathcal{G}}_{n}(\mathbf{x})=\left\{\tilde{\mathcal{G}}_{n}(\mathbf{x}), H_{T}\right\} \approx-\bar{N}(\mathbf{x}) n(\mathbf{x}) \tilde{\mathcal{G}}_{n}^{I I}(\mathbf{x})$.

On the other hand the time evolution of the constraint $\overline{\mathcal{R}}$ is equal to

$\partial_{t} \overline{\mathcal{R}}(\mathbf{x})=\left\{\overline{\mathcal{R}}(\mathbf{x}), H_{T}\right\} \approx \Gamma_{n} n(\mathbf{x}) \tilde{\mathcal{G}}_{n}^{I I}(\mathbf{x})=0$,

using (40), (46), and (51), together with the fact that $\Gamma^{i}=$ $\Gamma^{a b}=\Omega_{i}=0$. Now it is crucial to find a non-trivial solution of (73). In the case when $\tilde{\mathcal{G}}_{n}^{I I}$ were constant on the whole

\footnotetext{
${ }^{4}$ On general grounds we should expect that the given constraint is equivalent to the constraint derived in [47], when the canonical variables between these two formulations are properly identified.
}

phase space we would find that the only possible solution is $\Gamma_{n}=0$. Then from (72) we would also find $N=0$ and hence we should interpret $\overline{\mathcal{R}}$ together with $\tilde{\mathcal{G}}_{n}$ as second class constraints. However, this is a very unsatisfactory result, since it would imply the lack of the Hamiltonian constraint, while the theory is manifestly invariant under diagonal diffeomorphism. Fortunately $\tilde{\mathcal{G}}_{n}^{I I}$ depends on the phase space variables, so that it is more natural to obey (73) when we say that $\tilde{\mathcal{G}}_{n}^{I I}$ is an additional constraint imposed on the system.

Now with this interpretation we find that (72) vanishes on the constraint surface when $\tilde{\mathcal{G}}_{n}^{I I} \approx 0$. However, then we have to include the constraint $\tilde{\mathcal{G}}_{n}^{I I} \approx 0$ into the total Hamiltonian and analyze the stability of all constraints. Due to the fact that $\tilde{\mathcal{G}}_{n}^{I I}$ does not depend on $n$ we find that $p \approx 0$ is still a first class constraint. Now the time evolution of the constraint $\tilde{\mathcal{G}}_{n} \approx 0$ is equal to

$$
\begin{aligned}
\partial_{t} \tilde{\mathcal{G}}_{n}(\mathbf{x}) & =\left\{\tilde{\mathcal{G}}_{n}(\mathbf{x}), H_{T}\right\} \\
& \approx \int d^{3} \mathbf{y} \lambda^{I I}(\mathbf{y})\left\{\tilde{\mathcal{G}}_{n}(\mathbf{x}), \tilde{\mathcal{G}}_{n}^{I I}(\mathbf{y})\right\}=0,
\end{aligned}
$$

where $\lambda^{I I}$ is the Lagrange multiplier corresponding to the constraint $\tilde{\mathcal{G}}_{n}^{I I} \approx 0$. Since it can be shown that $\left\{\tilde{\mathcal{G}}_{n}(\mathbf{x}), \tilde{\mathcal{G}}_{n}^{I I}(\mathbf{y})\right\} \neq 0$ and it contains the derivative of the delta function we obtain the result that the only solutions of the equation above is $\lambda^{I I}=0$. Then we also see that it is consistent to take the values of the Lagrange multipliers $\Gamma^{i}=\Gamma^{a b}=\Omega_{i}=0$ that were derived before the constraint $\tilde{\mathcal{G}}_{n}^{I I}$ was included into the total Hamiltonian. Then using $\lambda^{I I}=0$ we also find that the constraint $\overline{\mathcal{R}} \approx 0$ is preserved during the time evolution of the system. Further, the time evolution of the constraint $\tilde{\mathcal{G}}_{n}^{I I} \approx 0$ has the form

$$
\begin{aligned}
\partial_{t} \tilde{\mathcal{G}}_{n}^{I I}(\mathbf{x}) \approx & \left\{\tilde{\mathcal{G}}_{n}^{I I}(\mathbf{x}), \mathbf{T}_{T}(N)\right\} \\
& +\int d^{3} \mathbf{y} \Gamma_{n}(\mathbf{y})\left\{\tilde{\mathcal{G}}_{n}^{I I}(\mathbf{x}), \tilde{\mathcal{G}}_{n}(\mathbf{y})\right\}=0 .
\end{aligned}
$$

Generally this equation reduces to a complicated differential equation for $\Gamma_{n}$ and we presume that it can be solved, at least in principle. ${ }^{5}$

As the next step we will analyze the requirement of the preservation of the constraints $\mathcal{S}_{i}, \mathcal{T}_{a b}, \tilde{\mathcal{K}}^{i}$, which, however, simplifies considerably due to the fact that $\Gamma^{i}=\Gamma^{a b}=$

\footnotetext{
5 There is one important subtlety with the given arguments, which is the possibility of the existence of the non-trivial solution of (74) due to the presence of the partial derivatives that are presented in the given equation. However, the existence of a given solution would imply the existence of an additional gauge symmetry, which, however, is not expected here. We believe that there is no such solution even if we were not able to show this explicitly. In light of these complicated results we mean that it is remarkable how efficient is the Hamiltonian analysis of the bimetric theory of gravity presented in [47].
} 
$\Omega_{i}=0$. We start with the constraint $\mathcal{S}_{i}$

$\partial_{t} \mathcal{S}_{i}=\left\{\mathcal{S}_{i}, H_{T}\right\} \approx \int d^{3} \mathbf{x} \bar{N}(\mathbf{x})\left\{\mathcal{S}_{i}, \overline{\mathcal{R}}(\mathbf{x})\right\}+f_{i}{ }^{a} v_{a}=0$,

using also the fact that $\left\{\mathcal{S}_{i}(\mathbf{x}), L_{a b}^{\mathrm{br}}(\mathbf{y})\right\}=0$. Now due to the fact that the matrix $f_{i}^{a}$ is non-singular we find that this equation can be solved for $v_{a}$.

In the case of the constraints $\mathcal{T}_{a b}$ we find

$$
\begin{aligned}
\partial_{t} \mathcal{T}_{a b}=\left\{\mathcal{T}_{a b}, H_{T}\right\} \approx & \int d^{3} \mathbf{x} \bar{N}(\mathbf{x})\left\{\mathcal{T}_{a b}, \overline{\mathcal{R}}(\mathbf{x})\right\} \\
& +\triangle_{\mathcal{T}_{a b}, k^{c}} v_{c}+\triangle_{\mathcal{T}_{a b}, L_{c d}^{\text {br }}} v^{c d}=0,
\end{aligned}
$$

where the matrix $\Delta_{\mathcal{T}_{a b}, k^{c}}$ is defined as

$$
\left\{\mathcal{T}_{a b}(\mathbf{x}), k^{c}(\mathbf{y})\right\} \equiv \triangle_{\mathcal{T}_{a b}, k^{c}}(\mathbf{x}) \delta(\mathbf{x}-\mathbf{y})
$$

Now using the fact that the matrix $\triangle_{\mathcal{T}_{a b}, L_{c d}^{\text {br }}}$ is non-singular and that $v_{a}$ were determined by (76) we find that (77) can be explicitly solved for $v^{a b}$.

Finally we proceed to the analysis of the equation of motion of the constraint $\tilde{\mathcal{K}}^{i}$,

$$
\begin{aligned}
\partial_{t} \tilde{\mathcal{K}}^{i}= & \left\{\tilde{\mathcal{K}}^{i}, H_{T}\right\} \approx \int d^{3} \mathbf{x} \bar{N}(\mathbf{x})\left\{\tilde{\mathcal{K}}^{i}, \overline{\mathcal{R}}(\mathbf{x})\right\}+v^{n} \triangle_{\tilde{\mathcal{K}}^{i}, p} \\
& +v^{a b} \triangle_{\tilde{\mathcal{K}}^{i}, L_{a b}^{\mathrm{br}}}+v^{i}+v_{c} \triangle_{\tilde{\mathcal{K}}^{i}, k^{c}}=0
\end{aligned}
$$

where we defined

$$
\begin{aligned}
& \left\{\tilde{\mathcal{K}}^{i}(\mathbf{x}), p(\mathbf{y})\right\}=\left[-\frac{1}{\gamma n^{2}} f_{a}^{i} p^{a}+\frac{1}{\gamma} e^{i b} p_{b}\right] \delta(\mathbf{x}-\mathbf{y}) \\
& \equiv \triangle_{\tilde{\mathcal{K}}^{i}, p}(\mathbf{x}) \delta(\mathbf{x}-\mathbf{y}) \text {, } \\
& \left\{\tilde{\mathcal{K}}^{i}(\mathbf{x}), L_{a b}^{\mathrm{br}}(\mathbf{y})\right\}=\frac{2 n}{\gamma}\left(e^{i}{ }_{a} p_{b}-e^{i}{ }_{b} p_{a}\right)(\mathbf{x}) \delta(\mathbf{x}-\mathbf{y}) \\
& \equiv \triangle_{\tilde{\mathcal{K}}^{i}, L_{a b}^{\mathrm{br}}} \delta(\mathbf{x}-\mathbf{y}), \\
& \left\{\tilde{\mathcal{K}}^{i}(\mathbf{x}), k^{c}(\mathbf{y})\right\}=\frac{1}{\gamma^{2}} p^{c}\left(\tilde{\mathcal{K}}^{i}-n^{i}\right) \delta(\mathbf{x}-\mathbf{y}) \\
& -\frac{1}{\gamma}\left(\frac{1}{n} f_{c}^{i}+n e_{c}^{i}\right) \delta(\mathbf{x}-\mathbf{y}) \\
& \approx-\left(\frac{1}{\gamma^{2}} p^{c} n^{i}+\frac{1}{\gamma} \frac{1}{n} f_{c}^{i}+\frac{n}{\gamma} e_{c}^{i}\right) \\
& \times \delta(\mathbf{x}-\mathbf{y}) \equiv \triangle_{\tilde{\mathcal{K}}^{i}, k^{c}}(\mathbf{x}) \delta(\mathbf{x}-\mathbf{y}) \text {. }
\end{aligned}
$$

We see that (79) can be solved for $v^{i}$ knowing the Lagrange multipliers $v_{a b}, v_{c}, v_{n}$. Note that $v_{n}$ is still undetermined, which is a reflection of the fact that $p \approx 0$ is a first class constraint.

Let us outline our results and determine the physical degrees of freedom of the given theory. We have $N_{\text {f.c.c. }}=12$ first class constraints $\overline{\mathcal{R}}, \overline{\mathcal{R}}_{i}, \bar{P}, P_{i}, L_{a b}^{\text {diag }}, p{ }^{6}$

\footnotetext{
${ }^{6}$ Strictly speaking, we should include the appropriate combinations of the second class constraints listed below them in order to ensure that the constraints $\overline{\mathcal{R}}, \overline{\mathcal{R}}_{i}$ have vanishing Poisson brackets with all constraints.
}

Then we have $N_{\text {s.c.c. }}=20$ second class constraints $p_{i}, k^{a}, L_{a b}^{\mathrm{br}}, \tilde{\mathcal{G}}_{n}, \tilde{\mathcal{G}}_{n}^{I I}, \mathcal{S}_{i}, \tilde{\mathcal{K}}^{i}, \mathcal{T}_{a b}$. We also have $N_{\text {ph.s.d.f. }}=$ 58 phase space degrees of freedom $\bar{N}, \bar{P}, \bar{N}^{i}, P_{i}, n, p, n^{i}$, $p_{i}, p_{a}, k^{a}, e_{i}^{a}, \pi_{a}^{i}, f_{i}^{a}, \rho_{a}^{i}$. Then the number of physical degrees of freedom $N_{\text {p.d.f. }}$ is [39]

$N_{\text {p.d.f. }}=\frac{1}{2}\left(N_{\text {ph.s.d.f. }}-2 N_{\text {f.c.c. }}-N_{\text {s.c.c. }}\right)=7$,

which could be interpreted as two physical degrees of freedom of the massless graviton, five physical degrees of freedom corresponding to the massive graviton. In other words we have shown that the bigravity in the vierbein formulation is ghost free.

\section{Conclusion}

This paper was devoted to the Hamiltonian analysis of the bimetric theory of gravity in the form introduced in [8]. We found the corresponding Hamiltonian and determined the primary constraints of the theory. Then we analyzed the requirement of the preservation of these constraints and we determined the corresponding secondary constraints. Finally, we determined the conditions when these constraints are preserved and we found that there is an additional constraint. As a result the constraint structure of the given theory suggests that this theory is free of ghosts.

However, it is still important to stress that, even if the nonlinear massive gravity is ghost free, this does not mean that the given theory is consistent. In fact, it was shown that nonlinear massive gravity suffers from the superluminality in its decoupling limit [16-18]. It was also shown that generally it contains tachyonic modes [19,20]. Further, the analysis of the cosmological properties of non-linear massive gravity showed that it exhibits the ghost instabilities about its homogeneous solutions [21-23]; see also $[42,43] .{ }^{7}$ On the other hand it was shown very recently in [48] that a non-linear bimetric theory of gravity could lead to viable cosmology under some conditions. In fact, the bimetric theory of gravity has an important advantage with respect to non-linear massive gravity where the second metric is not fixed by hand but is dynamical as well. Clearly a bimetric theory of gravity is a very promising generalization of gravity that deserves to be studied further.

\section{Footnote 6 continued}

However, these corrected constraints coincide with the original one when the second class constraints strongly vanish, where, however, we have to replace Poisson brackets between phase space variables with corresponding Dirac brackets.

${ }^{7}$ However, quite recently the improved version of non-linear massive gravity was proposed in [24], which is claimed to be unitary with all degrees of freedom propagating on a homogeneous, isotropic and selfaccelerating de Sitter background. 
Acknowledgments I would like to thank S. Alexandrov for very useful discussions and for his finding of a crucial error in the first version of this paper. This work was supported by the Grant agency of the Czech republic under the grant P201/12/G028.

Open Access This article is distributed under the terms of the Creative Commons Attribution License which permits any use, distribution, and reproduction in any medium, provided the original author(s) and the source are credited.

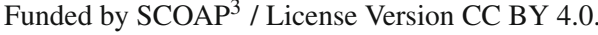

\section{Appendix A: Hamiltonian formalism of general relativity in vierbein formulaton}

In this appendix we perform the Hamiltonian formalism of the general relativity in vierbein formulation. We mostly follow [28-31].

Let us consider the general relativity Lagrangian density written in the form

$$
\begin{aligned}
\mathcal{L}= & M_{p}^{2} \operatorname{det} E\left(\Omega_{A}^{A B} \Omega_{C B}^{C}-\frac{1}{2} \Omega^{C A B} \Omega_{C B A}\right. \\
& \left.-\frac{1}{4} \Omega^{B A C} \Omega_{B A C}\right),
\end{aligned}
$$

where

$\Omega^{C A B}=E^{\mu C} E^{\nu A} \partial_{[\mu} E_{\nu]}^{B}, E^{\mu A}=E_{B}^{\mu} \eta^{B A}$,

and where $\mu, v, \cdots=0,1,2,3$ and where $A, B, \cdots=$ $0,1,2,3$. Note that by definition we have two covariant derivatives $\hat{\mathcal{D}}_{\mu}$ and $\hat{\nabla}_{\mu}$. $\hat{\mathcal{D}}_{\mu}$ is covariant with respect to both general coordinate transformations in spacetime as well as local Lorentz transformations on the flat index, while $\hat{\nabla}_{\mu}$ is covariant under general coordinate transformations. We have

$\hat{\mathcal{D}}_{\mu} \lambda^{\nu A}=\partial_{\mu} \lambda^{\nu A}+\hat{\Gamma}_{\mu \gamma}^{\nu} \lambda^{\gamma A}+\hat{\omega}_{\mu B}^{A} \lambda^{\nu B}$,

$\hat{\nabla}_{\mu} \lambda^{\nu A}=\partial_{\mu} \lambda^{\nu A}+\hat{\Gamma}_{\alpha \gamma}^{\nu} \lambda^{\gamma A}$.

We require that these covariant derivatives are compatible with the vierbein and the metric

$\hat{\mathcal{D}}_{\mu} E_{v}{ }^{A}=0$,

$\hat{\nabla}_{\mu} \hat{g}_{\nu \sigma}=\hat{\mathcal{D}}_{\mu} \hat{g}_{\nu \sigma}=0$.

where $\hat{g}_{\mu \nu}=E_{\mu}^{A} E_{\nu}{ }^{B} \eta_{A B}$. Note that from (85) we obtain

$\hat{\nabla}_{\mu} E_{A}^{v}=-\hat{\omega}_{\mu A}{ }^{B} E_{B}^{v}$.

From (85) and requiring $\hat{\Gamma}_{[\mu \nu]}^{\rho}=0$ it is possible to uniquely determine $\hat{\Gamma}_{\mu \nu}^{\rho}$ and $\hat{\omega}_{\mu B}^{A}$ as functions of the vierbein $E_{\mu}{ }^{A}$. Explicitly, the first equation in (85) can be solved as

$\hat{\omega}_{\mu}^{A B}=\frac{1}{2} e_{\mu C}\left(\Omega^{C A B}+\Omega^{B C A}-\Omega^{A B C}\right)$.
Let us now consider following $3+1$ decomposition of the tetrad:

$$
\begin{aligned}
E_{0}{ }^{A}= & N N^{A}+N^{a} V_{a}{ }^{A}, E_{i}{ }^{A}=V_{i}{ }^{A}, N^{B} \eta_{A B} V_{i}{ }^{B}=0, \\
& N^{A} \eta_{A B} N^{B}=-1,
\end{aligned}
$$

where $i, j, k, \cdots=1,2,3$ and $a, b, c, \cdots=1,2,3$. The inverse vielbein obeys

$E_{B}^{\mu} E_{\mu}^{C}=\delta_{B}^{C}, E_{A}^{\mu} E_{v}{ }^{A}=\delta^{\mu}{ }_{v}$.

Using this decomposition it is rather straightforward to perform the Legendre transform using this decomposition. However, it is more convenient to partly break the manifest Lorentz invariance in such a way that the vierbein takes the upper triangular form (1). In this case we identify $V_{i}{ }^{a}$ with $e_{i}^{a}$ where $e_{i}^{a}$ defines the three dimensional metric $g_{i j}=e_{i}^{a} e_{j}^{b} \delta_{a b}$.

Now using (88) and also the partial gauge fixing we obtain the following decomposition of $\Omega^{A B C}$ :

$\Omega_{a}^{0 b}=\frac{1}{N} e_{a}^{i}\left(\partial_{0} e_{i}^{b}-N^{j} \partial_{j} e_{i}^{b}-e_{a}^{i} e_{j}^{b} \partial_{i} N^{j}\right)$,

$\Omega_{a}^{00}=\frac{1}{N} e_{a}^{i} \partial_{i} N$

$\Omega^{a}{ }^{0}=0$,

$\Omega_{a b}^{c}=e_{a}^{i} e_{b}^{j}\left(\partial_{i} e_{j}^{c}-\partial_{j} e_{i}^{c}\right)$.

The general relativity Lagrangian now takes the form

$$
\begin{aligned}
\mathcal{L}= & M_{P}^{2} N e\left(-\frac{1}{2} \Omega^{0(a b)} \Omega_{0 a b}+\Omega_{a}^{0 a} \Omega_{0 b}^{b}+2 \Omega_{0}^{a}{ }_{0}^{0} \Omega_{a b} b\right. \\
& \left.-\frac{1}{4} \Omega^{a b c} \Omega_{a b c}-\frac{1}{2} \Omega^{a b c} \Omega_{a c b}+\Omega_{c}^{a c} \Omega_{b a}^{b}\right),
\end{aligned}
$$

where $e=\operatorname{det} e_{i}^{a}$ and where we have the following convention:

$X^{(a b)}=X^{a b}+X^{b a}, X^{(a b)} X_{(a b)}=2 X^{(a b)} X_{b a}$.

Note that we can write

$$
\begin{aligned}
N e^{(3)} R= & N e\left(\Omega_{b}^{a b} \Omega_{c a}^{c}-\frac{1}{4} \Omega^{a b c} \Omega_{a b c}-\frac{1}{2} \Omega^{a b c} \Omega_{a c b}\right) \\
& +\nabla_{[i}\left(N e e^{j a} \nabla_{j]} e_{a}^{i}\right)-2 e e^{i}{ }_{a} \partial_{[i} e_{j]}^{a} g^{j k} \partial_{k} N,
\end{aligned}
$$

where $\nabla_{i}$ is a covariant derivative compatible with $g_{i j}$ so that $\nabla_{i} g_{j k}=0$. Then neglecting the surface term we find that (91) has a form that is suitable for the Hamiltonian formulation,

$$
\mathcal{L}=M_{g}^{2} N e\left(-\frac{1}{2} \Omega^{0 a b} \Omega_{0(a b)}+\Omega^{0 a}{ }_{a}^{a} \Omega_{0 b}^{b}+{ }^{(3)} R\right),
$$

where

$$
\Omega^{0 a b}=\delta^{a c} \Omega_{c}^{0 b}, \quad \Omega_{0 a b}=-\Omega_{b}^{0 d} \delta_{d c}, \quad \Omega_{0 b}^{b}=-\Omega_{b}^{0 b} .
$$


Note that (92) implies

$$
\Omega^{0(a b)} \Omega_{0 a b}=\frac{1}{2} \Omega^{0(a b)} \Omega_{0(a b)} .
$$

Then from (94) we find the momenta $\pi_{a}^{i}$ conjugate to $e_{i}{ }^{a}$

$\pi_{a}^{i}=\frac{\delta \mathcal{L}}{\delta \partial_{0} e_{i}^{a}}=M_{P}^{2} e\left(e_{c}^{i} \Omega^{0(c d)} \delta_{d a}-2 \Omega_{b}^{0 b} e_{a}^{i}\right)$,

so that

$\Omega_{a}^{0 a}=-\frac{1}{4 e} e_{i}^{a} \pi_{a}^{i}$

Then it is easy to express $\Omega^{0(a b)}$ as a function of $\pi_{a}^{i}$ and $e_{i}^{a}$,

$\Omega^{0(a b)}=\frac{1}{e}\left(e_{i}^{a} \pi^{i}{ }_{c} \delta^{c b}-\frac{1}{2} \delta^{a b} e_{i}{ }^{c} \pi^{i}{ }_{c}\right)$

Using this result we easily find the Hamiltonian from (94) and hence the corresponding Hamiltonian

$\mathcal{H}=N \mathcal{R}+N^{i} \mathcal{R}_{i}$

where

$$
\begin{aligned}
& \mathcal{R}=\frac{1}{4 M_{g}^{2} e}\left(e_{i}{ }^{a} \pi^{i}{ }_{c} \delta^{c g} \delta_{a e} e_{j}{ }_{j} \pi_{g}^{j}-\frac{1}{2}\left(e_{i}{ }^{a} \pi^{i}{ }_{a}\right)^{2}\right)-M_{g}^{2} e^{(3)} R, \\
& \mathcal{R}_{i}=-e_{i}{ }^{b} \mathcal{D}_{j} \pi^{j}{ }_{b},
\end{aligned}
$$

where $\mathcal{D}_{i}$ is the covariant derivative compatible with $e_{i}^{a}$,

$\mathcal{D}_{i} e_{j}^{a}=0$

Note also that we neglected the total derivative terms in the Hamiltonian (100). It is also important to stress that (97) implies the following primary constraints:

$L_{a b}=e_{i a} \pi_{b}^{i}-e_{i b} \pi_{a}^{i} \approx 0$.

By definition $L_{a b}$ are antisymmetric so that there are three constraints $L_{a b}$ in three dimensions.

To proceed further we need an explicit form of $\mathcal{D}_{i} \pi_{a}^{j}$. Since $\pi_{a}^{i}$ is a density of weight 1 we have ${ }^{8}$

$\mathcal{D}_{i} \pi_{a}^{j}=\partial_{i} \pi_{a}^{j}+\Gamma_{i k}^{j} \pi_{a}^{k}-\Gamma_{m i}^{m} \pi_{a}^{j}-\omega_{i}^{b}{ }_{a} \pi_{b}^{j}$,

so that

$\mathcal{D}_{i} \pi_{a}^{i}=\partial_{i} \pi_{a}^{i}+\omega_{i a}^{b} \pi_{b}^{i}$.

8 Note that the spin connection has the following prescription when it acts on an object with upper and lower Lorentz indices:

$\mathcal{D}_{i} X_{b}^{a}=\partial_{i} X_{b}^{a}+\omega_{i c}^{a} X_{b}^{c}-\omega_{i b}^{c} X_{c}^{a}$,
It is also convenient to introduce the notation

$\pi^{i j}=\frac{1}{4}\left(\pi_{a}^{i} e^{j a}+e^{i a} \pi_{a}^{j}\right)$,

which is similar to the notation used in [30]. Then it is easy to see that the Hamiltonian constraint $\mathcal{R}$ takes the familiar form,

$\mathcal{R}=\frac{1}{\sqrt{g} M_{g}^{2}} \mathcal{G}_{i j k l} \pi^{i j} \pi^{k l}-M_{g}^{2} \sqrt{g}^{(3)} R$,

where

$\mathcal{G}_{i j k l}=\frac{1}{2}\left(g_{i k} g_{j l}+g_{i l} g_{j k}-g_{i j} g_{k l}\right)$,

$\mathcal{G}^{i j k l}=\frac{1}{2}\left(g^{i k} g^{j l}+g^{i l} g^{j k}\right)-g^{i j} g^{k l}$,

which obey the relation

$\mathcal{G}_{i j k l} \mathcal{G}^{k l m n}=\frac{1}{2}\left(\delta_{i}^{m} \delta_{j}^{n}+\delta_{i}^{n} \delta_{j}^{m}\right)$.

Note also that in the same way we can write

$\mathcal{R}_{i}=-e_{i}^{a} \mathcal{D}_{j} \pi_{b}^{j}=-2 \nabla_{i} \pi^{i}{ }_{j}$,

using the fact that

$$
\begin{aligned}
-2 \nabla_{i} \pi_{j}^{i} & \approx-\nabla_{i} \pi_{a}^{i} e_{j}^{a}-\pi_{a}^{i} \nabla_{i} e_{j}^{a}= \\
& =-\nabla_{i} \pi_{a}^{i} a_{j}^{a}+\pi_{a}^{i} \omega_{i b}^{a} e_{j}^{b}=-e_{i}^{a} \mathcal{D}_{j} \pi_{b}^{j},
\end{aligned}
$$

using also the fact that

$\pi_{j}^{i}=\pi^{i k} g_{k j}=\frac{1}{2} \pi_{a}^{i} e_{j}^{a}+L_{a d} e^{i a} e_{j}^{d}$.

By definition the canonical variables are $e_{i}{ }^{a}$ and $\pi_{b}^{j}$ with the following canonical Poisson brackets:

$\left\{e_{i}^{a}(\mathbf{x}), \pi_{b}^{j}(\mathbf{y})\right\}=\delta_{i}^{j} \delta_{b}^{a} \delta(\mathbf{x}-\mathbf{y})$

so that we obtain

$\left\{g_{i j}(\mathbf{x}), \pi^{k l}(\mathbf{y})\right\}=\frac{1}{2}\left(\delta_{i}^{k} \delta_{j}^{l}+\delta_{i}^{l} \delta_{j}^{k}\right) \delta(\mathbf{x}-\mathbf{y})$.

On the other hand from (107) and from (114) we find

$$
\begin{aligned}
\left\{\pi^{i j}(\mathbf{x}), \pi^{k l}(\mathbf{y})\right\}= & \frac{1}{16}\left(g^{i l} L^{k j}+g^{j l} L^{k i}+g^{j k} L^{l i}\right. \\
& \left.+g^{i k} L^{l j}\right) \delta(\mathbf{x}-\mathbf{y})=\mu^{i j k l} \delta(\mathbf{x}-\mathbf{y}) .
\end{aligned}
$$

This result implies that there are additional terms when we calculate the Poisson brackets between the constraints as was 
in a nice way shown in [30]. More precisely, let us introduce the smeared form of the constraints $\mathcal{R}, \mathcal{R}_{i}$ and $L_{a b}$

$$
\begin{gathered}
\mathbf{T}_{T}(N)=\int d^{3} \mathbf{x} N \mathcal{R}, \mathbf{T}_{S}\left(N^{i}\right)=\int d^{3} \mathbf{x} N^{i} \mathcal{R}_{i}, \\
\mathbf{L}\left(N^{a b}\right)=\int d^{3} \mathbf{x} N^{a b} L_{a b} .
\end{gathered}
$$

Then, following [30] we find

$$
\begin{aligned}
\left\{\mathbf{T}_{T}(N), \mathbf{T}_{T}(M)\right\}= & \mathbf{T}_{S}\left(\left(N \partial_{i} M-M \partial_{i} N\right) g^{i j}\right), \\
\left\{\mathbf{T}_{S}\left(N^{i}\right), \mathbf{T}_{T}(M)\right\}= & \mathbf{T}_{T}\left(N^{i} \partial_{i} M\right)+\int d^{3} \mathbf{x} \nabla_{j} N^{i} \lambda_{i}{ }^{j} M, \\
\left\{\mathbf{T}_{S}\left(N^{i}\right), \mathbf{T}_{S}\left(M^{j}\right)\right\}= & \mathbf{T}_{S}\left(\left(N^{i} \partial_{i} M^{j}-M^{j} \partial_{i} N^{j}\right)\right) \\
& +\int d^{3} \mathbf{x} \nabla_{k} N^{i} \mu_{i}^{k}{ }_{j}^{l} \nabla_{l} M^{j}, \\
\left\{\mathbf{T}_{T}(N), \mathbf{L}\left(N^{a b}\right)\right\}= & 0,\left\{\mathbf{T}_{S}\left(N^{i}\right), \mathbf{L}\left(N^{a b}\right)\right\}=0, \\
\left\{L_{a b}(\mathbf{x}), L_{c d}(\mathbf{y})\right\}= & \left(\eta_{a d} L_{b c}+\eta_{b c} L_{a d}\right. \\
& \left.-\eta_{b d} L_{a c}-\eta_{a c} L_{b d}\right) \delta(\mathbf{x}-\mathbf{y}),
\end{aligned}
$$

where

$$
\begin{aligned}
\lambda^{i j} & =-4 \mu^{i j k l} K_{k l}=-\frac{1}{2}\left(K_{k}^{j} L^{i k}-K_{k}^{i} L^{j k}\right), \\
K_{i j} & =\frac{1}{\sqrt{g}}\left(\frac{1}{2} \pi^{m n} g_{n m} g_{i j}-\pi_{i j}\right) .
\end{aligned}
$$

We see that there are additional terms on the right side of the Poisson brackets between the constraints that are proportional to the primary constraints $L_{a b}$. These terms also vanish on the constraints surface. For that reason we will not write the explicit form of these terms in the calculations performed in the main body of the paper.

\section{References}

1. A. Salam, J.A. Strathdee, Nonlinear realizations. 1: the role of goldstone bosons. Phys. Rev. 184, 1750 (1969)

2. C.J. Isham, A. Salam, J.A. Strathdee, Spontaneous breakdown of conformal symmetry. Phys. Lett. B 31, 300 (1970)

3. M. Fierz, W. Pauli, On relativistic wave equations for particles of arbitrary spin in an electromagnetic field. Proc. R. Soc. Lond. A173, 211-232 (1939)

4. D.G. Boulware, S. Deser, Can gravitation have a finite range? Phys. Rev. D 6, 3368 (1972)

5. D.G. Boulware, S. Deser, Inconsistency of finite range gravitation. Phys. Lett. B 40, 227 (1972)

6. de Rham, C., Gabadadze, G., Tolley, A.J.: Resummation of massive gravity. Phys. Rev. Lett. 106, 231101 (2011). arXiv:1011.1232 [hep-th]

7. de Rham, C., Gabadadze, G., Tolley, A.J.: Ghost free massive gravity in the Stúckelberg language. Phys. Lett. B 711, 190 (2012). arXiv:1107.3820 [hep-th]

8. Hinterbichler, K., Rosen, R.A.: Interacting spin-2 fields. JHEP 1207, 047 (2012). arXiv:1203.5783 [hep-th]
9. Hassan, S.F., Schmidt-May, A., von Strauss, M.: Proof of consistency of nonlinear massive gravity in the Stúckelberg formulation. arXiv:1203.5283 [hep-th]

10. Hassan, S.F., Rosen, R.A.: Resolving the ghost problem in nonlinear massive gravity. Phys. Rev. Lett. 108, 041101 (2012). arXiv:1106.3344 [hep-th]

11. Hassan, S.F., Rosen, R.A.: On non-linear actions for massive gravity. JHEP 1107, 009 (2011). arXiv:1103.6055 [hep-th]

12. Hassan, S.F., Rosen, R.A.: Confirmation of the secondary constraint and absence of ghost in massive gravity and bimetric gravity. JHEP 1204, 123 (2012). arXiv:1111.2070 [hep-th]

13. Kluson, J., Non-linear massive gravity with additional primary constraint and absence of ghosts. Phys. Rev. D 86, 044024 (2012). arXiv:1204.2957 [hep-th]

14. Hassan, S.F., Rosen, R.A., Schmidt-May, A.: Ghost-free massive gravity with a general reference metric. JHEP 1202, 026 (2012). arXiv:1109.3230 [hep-th]

15. Hassan, S.F., Rosen, R.A.: Bimetric gravity from ghost-free massive gravity. JHEP 1202, 126 (2012). arXiv:1109.3515 [hep-th]

16. Gruzinov, A.: All Fierz-Paulian massive gravity theories have ghosts or superluminal modes. arXiv:1106.3972 [hep-th]

17. Burrage, C., de Rham, C., Heisenberg, L., Tolley, A.J.: Chronology protection in Galileon models and massive gravity. JCAP 1207, 004 (2012). arXiv:1111.5549 [hep-th]

18. de Fromont, P., de Rham, C., Heisenberg, L., Matas, A.: Superluminality in the Bi- and multi-Galileon. arXiv:1303.0274 [hep-th]

19. Deser, S., Waldron, A.: Inconsistencies of massive charged gravitating higher spins. Nucl. Phys. B 631, 369 (2002). [hep-th/0112182]

20. Deser, S., Sandora, M., Waldron, A.: Nonlinear partially massless from massive gravity? Phys. Rev. D 87, 101501(R) (2013). arXiv:1301.5621 [hep-th]

21. De Felice, A., Gumrukcuoglu, A.E., Mukohyama, S.: Massive gravity: nonlinear instability of the homogeneous and isotropic universe. Phys. Rev. Lett. 109, 171101 (2012). arXiv:1206.2080 [hep-th]

22. De Felice, A., Gmrkog(lu), A.E., Lin, C., Mukohyama, S.: Nonlinear stability of cosmological solutions in massive gravity. JCAP 1305, 035 (2013) arXiv:1303.4154 [hep-th]

23. De Felice, A., Gumrukcuoglu, A.E., Lin, C., Mukohyama, S.: On the cosmology of massive gravity. arXiv:1304.0484 [hep-th]

24. De Felice, A., Mukohyama, S.: Towards consistent extension of quasidilaton massive gravity. arXiv:1306.5502 [hep-th]

25. Gourgoulhon, E.: $3+1$ formalism and bases of numerical relativity. gr-qc/0703035 [GR-QC]

26. Arnowitt, R.L., deser, S., Misner, C.W.: The dynamics of general relativity. [gr-qc/0405109]

27. Chaichian, M., Oksanen, M., Tureanu, A.: Arnowitt-Deser-Misner representation and Hamiltonian analysis of covariant renormalizable gravity. Eur. Phys. J. C71, 1657 (2011) [Erratum-ibid. C 71 (2011) 1736]. arXiv:1101.2843 [gr-qc]

28. Peldan, P.: Actions for gravity, with generalizations: a review. Class. Quant. Grav. 11, 1087 (1994). [gr-qc/9305011]

29. H. Nicolai, H.J. Matschull, Aspects of canonical gravity and supergravity. J. Geom. Phys. 11, 15 (1993)

30. M. Henneaux, Poisson brackets of the constraints in the Hamiltonian formulation of tetrad gravity. Phys. Rev. D 27, 986 (1983)

31. J.M. Charap, M. Henneaux, J.E. Nelson, Explicit form of the constraint algebra in tetrad gravity. Class. Quant. Grav. 5, 1405 (1988)

32. Yepez, J.: Einstein's vierbein field theory of curved space. arXiv:1106.2037 [gr-qc]

33. Damour, T., Kogan, I.I.: Effective Lagrangians and universality classes of nonlinear bigravity. Phys. Rev. D 66, 104024 (2002). [hep-th/0206042]

34. Kluson, J.: Is bimetric gravity really ghost free?. arXiv:1301.3296 [hep-th] 
35. Kluson, J.: Hamiltonian formalism of general bimetric gravity. arXiv:1303.1652 [hep-th]

36. Kluson, J.: Hamiltonian formalism of particular bimetric gravity model. arXiv:1211.6267 [hep-th]

37. Soloviev, V.O., Tchichikina, M.V.: Bigravity in Kuchar's Hamiltonian formalism. 1. The general case. arXiv:1211.6530 [hep-th]

38. Soloviev, V.O., Tchichikina, M.V.: Bigravity in Kuchar's Hamiltonian formalism. 2. The special case. arXiv:1302.5096 [hep-th]

39. M. Henneaux, C. Teitelboim, Quantization of gauge systems (Princeton University Press, Princeton, 1992)

40. Comelli, D., Nesti, F., Pilo, L.: Massive gravity: a general analysis. arXiv:1305.0236 [hep-th]

41. Comelli, D., Nesti, F., Pilo, L.: Weak massive gravity. arXiv: 1302.4447 [hep-th]

42. Berezhiani, L., Chkareuli, G., Gabadadze, G.: Restricted galileons. arXiv:1302.0549 [hep-th]
43. Berezhiani, L., Chkareuli, G., de Rham, C., Gabadadze, G., Tolley, A.J.: Mixed galileons and spherically symmetric solutions. arXiv:1305.0271 [hep-th]

44. A.H. Chamseddine, A. Salam, J.A. Strathdee, Strong gravity and supersymmetry. Nucl. Phys. B 136, 248 (1978)

45. Chamseddine, A.H.: Matrix gravity and massive colored gravitons. Phys. Rev. D 70, 084006 (2004). [hep-th/0406263]

46. Chamseddine, A.H.: Spontaneous symmetry breaking for massive spin two interacting with gravity. Phys. Lett. B 557, 247 (2003). [hep-th/0301014]

47. Alexandrov, S.: Canonical structure of tetrad bimetric gravity. Gen. Relat. Gravit. 46, 1639 (2014). arXiv:1308.6586 [hep-th]

48. De Felice, A., Gumrukcuoglu, A.E., Mukohyama, S., Tanahashi, N., Tanaka, T.: Viable cosmology in bimetric theory. arXiv: 1404.0008 [hep-th] 